\title{
Synthesis, Characterization and Application of Poly(4-vinylpyridine)- Supported Brønsted Acid as Reusable Catalyst for Acetylation Reaction
}

\author{
Kalyan Jyoti Borah, Papia Dutta, and Ruli Borah*
}

\author{
Chemical Sciences Department, Tezpur University, Napaam-784028,Tezpur-Assam, India. *E-mail: ruli@tezu.ernet.in \\ Received October 15, 2010, Accepted November 15, 2010
}

\begin{abstract}
Poly(4-vinylpyridine)-supported Brønsted acids (P4VP-HX) were prepared by wet impregnation technique. These supported acids were found as efficient heterogeneous green catalysts for acetylation of alcohol, amine and phenol with different catalytic activities. The wide application of P4VP-HX as reusable solid acid catalyst in organic reactions is possible because of its simple preparation and handling, stability, simple work up procedure.
\end{abstract}

Key Words: Heterogeneous, Polymer support, Reusable, Solid acid

\section{Introduction}

In recent years, the efficient use of non-toxic and more selective supported solid acidic ${ }^{1}$ catalyst has received more attention in different areas of organic synthesis because of their environmentally compatibility, reusability, high selectivity, simple operation and ease of isolation of the products. The study of polymer supported catalyst is motivated by the major advantages of the physical separation of the supported catalyst from the substrates and products, thereby allowing the recycling of expensive or toxic catalyst and thus releases less waste material to the environment. The potential use of polymer supported catalysts as heterogeneous and recyclable catalysts in organic transformation make reaction methods more convenient, economic and environmentally benign. The reactions can be performed under mild conditions and product purification is simple due to easy separation of insoluble polymer support from the reaction medium. Many reactions ${ }^{2}$ can be carried out cleanly, rapidly and in high yields. Generally, polystyrene-based solid acidic catalysts show relatively low activity and the activity of the catalyst decreases due to insufficient stability of the aromatic ring of the polymer and slow diffusion of substrate into the polymer network. Moreover, the actual loading is limited by Lewis acid catalyzed cross linking of chloromethyl groups within the polystyrene-based resin. The best effective method to increase the maximal loading of the carrier material is obtained by using low molecular weight functional monomers. ${ }^{3}$ Poly(4vinylpyridine) is an interesting material because of its stable pyridyl group and ability to form charge transfer complex with acidic dopants. ${ }^{4}$ It is widely used universal surface modifier for immobilization ${ }^{5}$ of metal triflates, ruthenates, and nanoparticles. Mineral acids such as $\mathrm{H}_{2} \mathrm{SO}_{4}, \mathrm{HCl}, \mathrm{H}_{3} \mathrm{PO}_{4}$ are widely used as catalyst in several organic syntheses. ${ }^{6}$ But direct use of the mineral acid in liquid state is not favorable because of vigorous reaction condition, corrosive nature, toxicity, and hygroscopic nature, difficulty in separation, recovery and recycling. Poly(4vinylpyridine) seems to an attractive support to immobilize mineral acids because of the basic nature of pyridyl group. Herein, we report the successful synthesis of P4VP supported Brønsted acid catalyst (P4VP-HX) in ether solution at room

temperature, their characterization and applications as simple reusable solid acid catalysts in acetylation of $-\mathrm{OH}$ and $-\mathrm{NH}_{2}$ functionality.

\section{Experimental}

Materials. Poly(4-vinylpyridine), cross linked with 2\% DVB, was purchased from ACROS ORGANICS. Reagent grade sulphuric acid, hydrochloric acid and phosphoric acid were used without further purification.

Preparation of Catalyst: P4VP-HX. Poly(4-vinylpyridine) $(500 \mathrm{mg})$ and the respective acids $\left(0.6 \mathrm{~mL}\right.$ of $\mathrm{H}_{2} \mathrm{SO}_{4}, \mathrm{HCl}$ and $\left.\mathrm{H}_{3} \mathrm{PO}_{4} \cdot 2 \mathrm{H}_{2} \mathrm{O}\right)$ were stirred in diethyl ether $(5 \mathrm{~mL})$ at room temperature for 15 minutes. Filtered and washed the solid catalyst with diethyl ether for three times. Finally, the catalysts dried at $70{ }^{\circ} \mathrm{C}$ for $2 \mathrm{hrs}$ and stored in vacuum decicator for use as catalyst.

Catalyst Characterization. FT-IR spectra of various catalyst samples were recorded on a Nicolet Impact Model-410 spectrometer with $1 \mathrm{~cm}^{-1}$ resolution within $400-4000 \mathrm{~cm}^{-1}$ region using the $\mathrm{KBr}$ pellet technique. The SEM analyses were carried out using a JEOL JSM-6390LV Scanning Electron Microscope equipped with an Energy-Dispersive X-ray analyzer (EDX). The images were taken at an accelerator voltage of $15 \mathrm{kV}$ and magnification of micrographs ranges from $\times 1000$ to $\times 2000$. Thermal stability of the supported catalysts was examined by SHIMADZU Thermo-gravimetric Analyzer-50 (TGA) in the temperature range of 20 to $600{ }^{\circ} \mathrm{C}$.

Catalyst Testing. We have examined the catalytic activity of the newly developed P4VP-HX system for the acylation of alcohols, phenols and amines in dichloromethane at room temperature (Scheme 1). The reactions were monitored by thin layer chromatography. The acetylated products were identified by comparison of their FT-IR and ${ }^{1} \mathrm{H}$ NMR spectroscopic data with those of authentic compounds (prepared by known method)

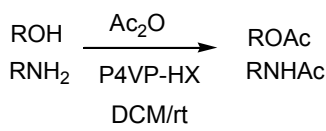

Scheme 1 
and literature reported data. ${ }^{7}$ Potential benefits of the P4VP-HX system include facilitating the easy separation of the catalyst from the reagents and reaction products and its recyclability for repeated use.

Typical Procedure for Acetylation of Alcohol. To a mixture of cholesterol $(1 \mathrm{mmol})$ and acetic anhydride $(3 \mathrm{mmol})$ in dichloromethane $(5 \mathrm{~mL}) \mathrm{P} 4 \mathrm{VP}-\mathrm{H}_{2} \mathrm{SO}_{4}(0.12 \mathrm{mmol})$ was added. After being stirred at room temperature for half an hour, the catalysts was removed by filtration and washed with dichloromethane $(10 \mathrm{~mL})$ for reuse. The filtrate was washed with aqueous sodium bicarbonate solution for removal of excess amount of acetic anhydride. Then the aqueous layer was extracted with dichloromethane $(10 \mathrm{~mL})$ to get the pure product. The solvent was evaporated under reduced pressure. The residue was pure enough for general purposes and further purification was achieved by thin layer chromatography to give analytically pure product (98\% yield).

\section{Result and Discussion}

Catalyst Characterization. In continuation of our research interest on the development of greener methodologies, ${ }^{8}$ initially, the different Brønsted acids were loaded on the polymer, poly (4-vinylpyridine), and cross linked with $2 \%$ divinyl benzene, (Fig. 1) following an incipient wet impregnation technique. Amount of acid group present in the polymeric chain was calculated based on the weight of supported polymer salt obtained

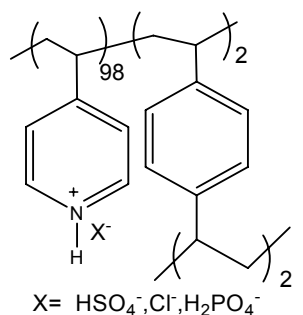

Figure 1. Structure of poly (4-vinylpyridine) supported acid catalyst (P4VP-HX).

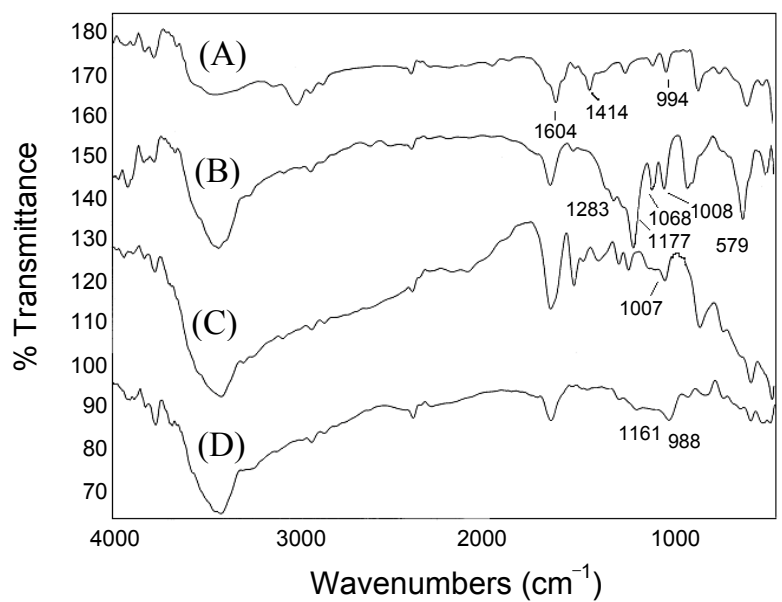

Figure 2. FT-IR spectrum of $\mathrm{P} 4 \mathrm{VP}(\mathrm{A}), \mathrm{P} 4 \mathrm{VP}-\mathrm{H}_{2} \mathrm{SO}_{4}$ (B), $\mathrm{P} 4 \mathrm{VP}-\mathrm{HCl}$ (C), and $\mathrm{P} 4 \mathrm{VP}-\mathrm{H}_{3} \mathrm{PO}_{4}(\mathrm{D})$. and the weight of poly(4-vinylpyridine) used. Amount of dopant present in poly(4-vinylpyridine) system such as $\mathrm{P} 4 \mathrm{VP}-\mathrm{H}_{2} \mathrm{SO}_{4}$, $\mathrm{P} 4 \mathrm{VP}-\mathrm{HCl}$, and $\mathrm{P} 4 \mathrm{VP}-\mathrm{H}_{3} \mathrm{PO}_{4}$ were found to be $0.652 \mathrm{~mol} \%$, $1.102 \mathrm{~mol} \%$ and $0.492 \mathrm{~mol} \%$ respectively. Higher amount of dopant was obtained in the case of hydrochloric acid. Characterizations of the polymer supported acid catalysts so formed were done by FT-IR, SEM, TGA and EDX studies.

FT-IR Spectra. The IR spectra of the P4VP and the supported catalysts (blends) are compared in Fig. 2. The pyridine rings exhibit strong $\mathrm{C}=\mathrm{C}$ stretching band at $1604 \mathrm{~cm}^{-1}$ and $\mathrm{C}=\mathrm{N}$ stretching absorption at $1414 \mathrm{~cm}^{-1}$. The monomer units of P4VP corresponding to the ring deformation appear around 993 - 994 $\mathrm{cm}^{-1}$, suffer a shift towards higher wave number, (1000 - 1008 $\mathrm{cm}^{-1}$ ) when the pyridine ring is protonated with Brønsted acid. This interaction increases the stiffness of the associated ring
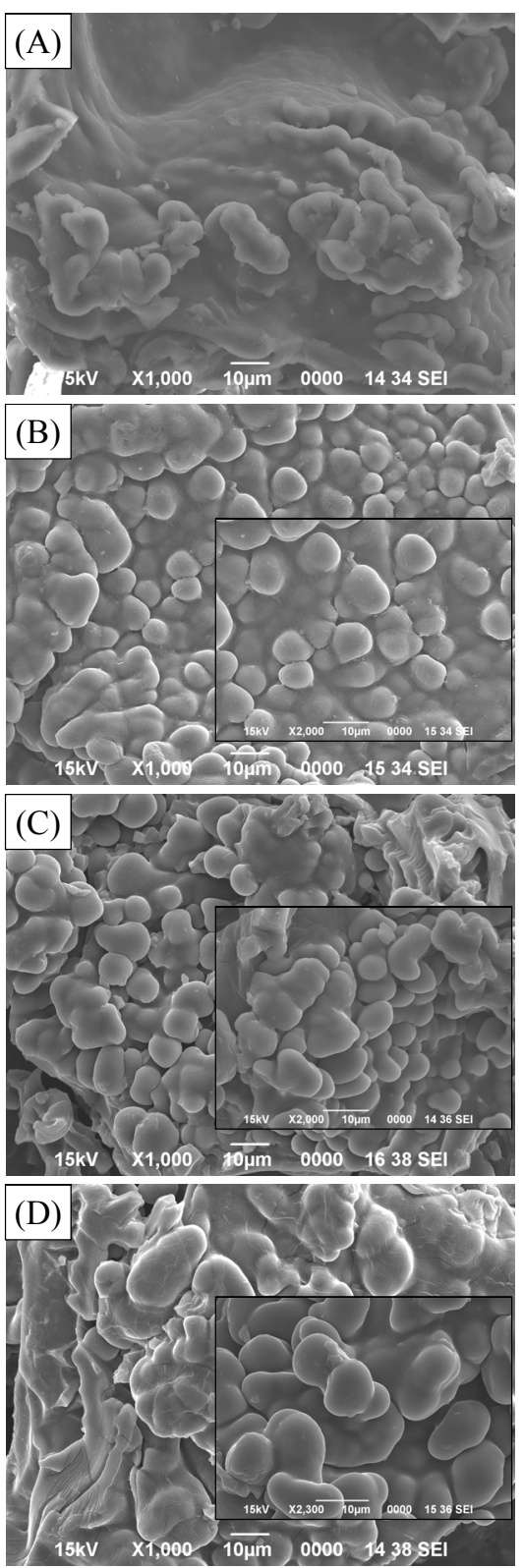

Figure 3. SEM photograph of P4VP and P4VP-HX $(\times 1000)$. SEM photograph of P4VP-HX $(\times 2000)$. 


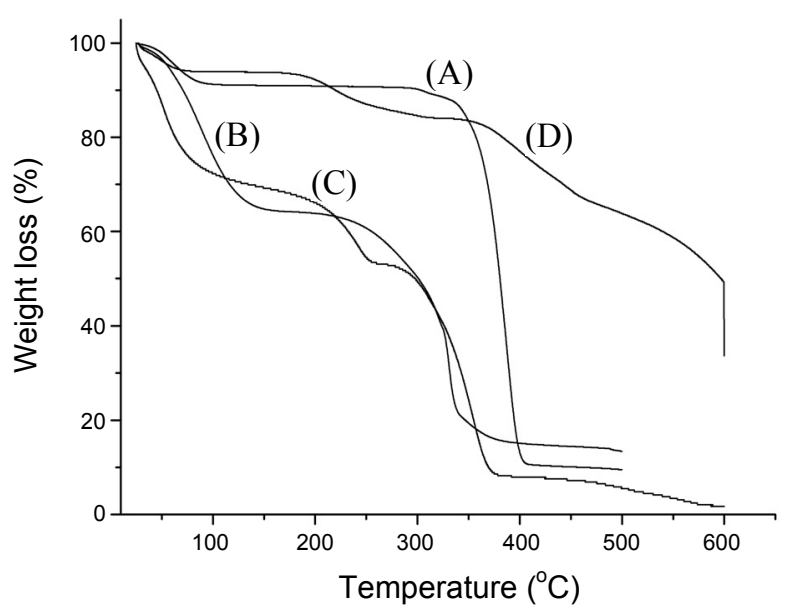

Figure 4. TGA curve of polymer (A) and supported acid catalysts (B, C, D).

and consequently more energy is required to deform the aromatic cycle, reflected in a higher wave number value. The P4VP has no $\mathrm{N}^{+}$---H stretching frequencies (Fig. 2A), but polymer salts $\mathrm{P} 4 \mathrm{VP}-\mathrm{H}_{2} \mathrm{SO}_{4}(\mathrm{~B}), \mathrm{P} 4 \mathrm{VP}-\mathrm{HCl}(\mathrm{C})$ and $\mathrm{P} 4 \mathrm{VP}-\mathrm{H}_{3} \mathrm{PO}_{4}$ (D) have this stretching vibrations at $3200-3400 \mathrm{~cm}^{-1}$ along with $\mathrm{O}-\mathrm{H}$ absorption. The polymer blend $\mathrm{B}$ indicates $\mathrm{v}_{\text {asym }} \mathrm{S}=\mathrm{O}$ at $1283-1177(\mathrm{vs}) \mathrm{cm}^{-1}$ and $\mathrm{v}_{\mathrm{sym}} \mathrm{S}=\mathrm{O} 1068 \mathrm{~cm}^{-1}$ respectively. The sharp band at $579 \mathrm{~cm}^{-1}$ is assigned to the $\mathrm{O}=\mathrm{S}=\mathrm{O}$ rocking band. ${ }^{9}$ Similarly, the polymer blend $\mathrm{D}$ shows an absorption band at $1161-988 \mathrm{~cm}^{-1}$ for the stretching of (P-O) $\mathrm{H}$.

SEM Characterization. The SEM of P4VP and other supported acid catalysts are shown in Fig. 3 (SEM photographs of A, $\mathrm{B}, \mathrm{C}$ and $\mathrm{D}$ at $\times 1000$ magnification). It is seen from the figure that the surface of the polymer is distinctly altered in each supported catalysts. No definite size particles are observed in case of P4VP (A). Sulfuric acid supported catalyst (B) particles are spherical with uniform morphology as compared to other two catalysts $(C, D)$. The SEM photograph of C and D indicates the presence of two types of particles spherical and elliptical. Major amounts of particles are elliptical in case of D (PVP-phosphoric acid). The surface of each particle is rough in case of $\mathrm{B}$ and smooth in case of C and D (Fig. 3 SEM photograph of B, C, and $\mathrm{D}$ at $\times 2000$ magnification).

Thermo Gravimetric Analysis. TGA analysis of the pure polymer (A) shows a one-step thermal decomposition with maximum decomposition temperature at $388^{\circ} \mathrm{C}$ (Fig. 4). The TGA curves of other supported polymers show two steps for $\mathrm{P} 4 \mathrm{VP}-\mathrm{H}_{2} \mathrm{SO}_{4}(\mathrm{~B})$ and three steps decompositions patterns for both $\mathrm{P} 4 \mathrm{VP}-\mathrm{HCl}(\mathrm{C})$ and $\mathrm{P} 4 \mathrm{VP}-\mathrm{H}_{3} \mathrm{PO}_{4}$ (D) catalysts. The stability of poly(4-vinylpyridine) decreases as shown by TGA curves (A, B \& C) when it formed complex with sulfuric acid and hydrochloric acid. The blend (D) with phosphoric acid shown higher stability up to $400{ }^{\circ} \mathrm{C}$ as compared to pure polymer (A). Above $50{ }^{\circ} \mathrm{C}$, a weight loss can be observed which can be attributed to the self condensation of phosphoric acid which ranges up to $350{ }^{\circ} \mathrm{C}$; however the loss rate doesn't correspond to the phosphoric acid content in the blends.

EDX Analysis. Energy dispersion X-ray (EDX) analyses of the catalysts were carried out for identification of Brønsted acids
(B)

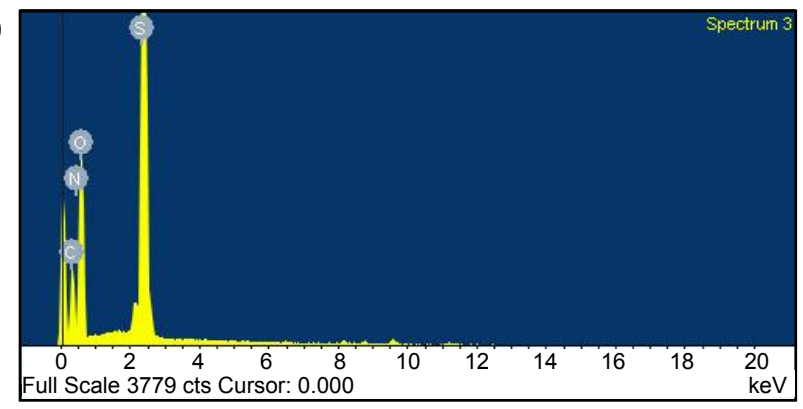

(C)

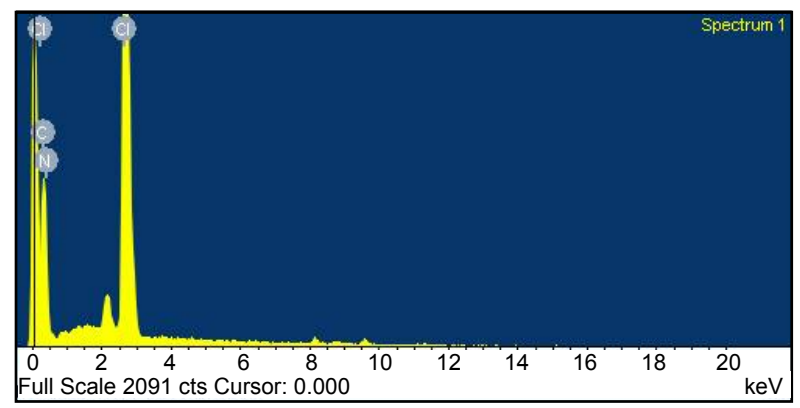

(D)

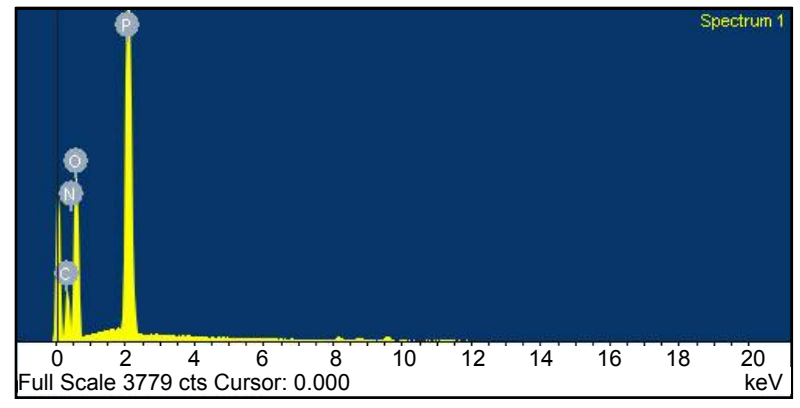

Figure 5. EDX pattern of supported acid catalysts (B, C, D).

on the surface of poly(4-vinylpyridine) support, as shown in Fig. 5 (B, C, D).

Acetylation Reactions Using Polymer Supported Acid Catalysts. The polymer supported acids were investigated as acetylating catalysts for the acylation of alcohols, phenols and amines in dichloromethane at room temperature (Scheme 1). The results are summarized in Table 1. The molar quantity of acetic anhydride is very important to the success of the acetylation reaction. With 1:1 and 1:2 ratios of cholesterol and acetic anhydride, the reactions were incomplete within 15 - $24 \mathrm{hrs}$ (entries $1,2)$ reaction periods. It was found that $1: 3$ ratio of alcohol and acetic anhydride decreases the reaction time to half an hour for cholesterol (entry 3 ). We have extended the same reaction conditions for other acetylation reactions. For polyhydroxy alcohol and phenols it yielded the fully acetylated products (entries 11, $16,17,18)$. Out of the three supported catalysts, $\mathrm{P} 4 \mathrm{VP}-\mathrm{H}_{2} \mathrm{SO}_{4}$ works better than $\mathrm{P} 4 \mathrm{VP}-\mathrm{HCl}$ and $\mathrm{P} 4 \mathrm{VP}-\mathrm{H}_{3} \mathrm{PO}_{4}$ in terms of reaction time and yields (entries 3, 6, 8, 9, 13, 16, 19). Primary (entries 8-10) and secondary alcohols (entries 3-7) can be easily acetylated at room temperature in dichloromethane using P4VP$\mathrm{H}_{2} \mathrm{SO}_{4}$ within short reaction period (8 - $35 \mathrm{~min}$ ) excluding tertiary alcohol (entry 22). Amino group can be preferentially acetylated in the presence of hydroxyl group (entry 20). Interestingly, phenolic -OH reacts slowly as compared to amino group (entries 13, 23). Monohydroxy phenolic compounds took longer 
Table 1. Acetylation of alcohols, phenols and amines with acetic anhydride catalyzed by P4VP supported acid catalysts

\begin{tabular}{|c|c|c|c|c|c|c|c|c|}
\hline & \multirow{2}{*}{ Substrate } & \multirow{2}{*}{$\begin{array}{l}\text { Ratio (Substrate: } \\
\text { acetic anhydride) }\end{array}$} & \multicolumn{2}{|c|}{$\mathrm{P} 4 \mathrm{VP}-\mathrm{H}_{2} \mathrm{SO}_{4}$} & \multicolumn{2}{|c|}{$\mathrm{P} 4 \mathrm{VP}-\mathrm{HCl}$} & \multicolumn{2}{|c|}{$\mathrm{P} 4 \mathrm{VP}-\mathrm{H}_{3} \mathrm{PO}_{4} \cdot 2 \mathrm{H}_{2} \mathrm{O}$} \\
\hline & & & Time (h) & yield $(\%)$ & Time (h) & yield (\%) & Time (h) & yield (\%) \\
\hline 1 & Chlolesterol & $1: 1$ & 24 & 50 & - & - & - & - \\
\hline 2 & Chlolesterol & $1: 2$ & 15 & 60 & - & - & - & - \\
\hline 3 & Cholesterol & $1: 3$ & 0.5 & 98 & 6 & 70 & 7 & 68 \\
\hline 4 & Diphenyl methanol & $1: 3$ & 0.5 & 96 & - & - & - & - \\
\hline 5 & Benzoin & $1: 3$ & $10 \mathrm{~min}$ & 95 & - & - & - & - \\
\hline 6 & Cyclohexanol & $1: 3$ & $8 \mathrm{~min}$ & 99 & $35 \mathrm{~min}$ & 97 & $40 \mathrm{~min}$ & 95 \\
\hline 7 & Methyl phenyl methanol & $1: 3$ & $35 \mathrm{~min}$ & 93 & - & - & - & - \\
\hline 8 & Octanol & $1: 3$ & $35 \mathrm{~min}$ & 94 & $80 \mathrm{~min}$ & 94 & 2 & 90 \\
\hline 9 & Cetyl alcohol & $1: 3$ & $20 \mathrm{~min}$ & 96 & 4 & 75 & 4 & 70 \\
\hline 10 & Benzyl alcohol & $1: 3$ & $15 \mathrm{~min}$ & 95 & - & - & - & - \\
\hline 11 & Ethylene Glycol & $1: 6$ & $1 \mathrm{~min}$ & 99 & - & - & - & - \\
\hline 12 & Allyl alcohol & $1: 3$ & 12 & NR & 12 & NR & 12 & NR \\
\hline 13 & Phenol & $1: 3$ & 1 & 95 & 7 & NR & 7 & NR \\
\hline 14 & 2-Naphthol & $1: 3$ & 1.5 & 87 & - & - & - & - \\
\hline 15 & 1-Naphthol & $1: 3$ & 3.5 & 85 & & & & \\
\hline 16 & Resorcinol & $1: 6$ & $1 \mathrm{~min}$ & 97 & 8 & 60 & 8 & 55 \\
\hline 17 & Catechol & $1: 6$ & $1 \mathrm{~min}$ & 97 & - & - & - & - \\
\hline 18 & Pyrrogalol & $1: 9$ & $1 \mathrm{~min}$ & 99 & - & - & - & - \\
\hline 19 & p-Cresol & $1: 3$ & $75 \min$ & 96 & 8 & NR & 8 & NR \\
\hline 20 & 4-Amino phenol & $1: 3$ & $1 \mathrm{~min}$ & 98 & - & - & - & - \\
\hline 21 & 4-Nitrophenol & $1: 3$ & 12 & NR & - & - & - & - \\
\hline 22 & $t$-Butyl alcohol & $1: 3$ & 3 & NR & - & - & - & - \\
\hline 23 & Aniline & $1: 3$ & $1 \mathrm{~min}$ & 95 & - & - & - & - \\
\hline
\end{tabular}

${ }^{a}$ All products were characterized by ${ }^{1}$ HNMR and also their TLC comparison with authentic sample prepared by reported method.

reaction time ( 1 - $3.5 \mathrm{~h})$ (entries 13-14) whereas polyhydroxy compounds completed the reaction within 1 min (entries 16-18) with $\mathrm{P} 4 \mathrm{VP}-\mathrm{H}_{2} \mathrm{SO}_{4}$. Allylic alcohol and nitro phenols are inactive for the reaction (entries 12, 21).

The catalysts $\mathrm{P} 4 \mathrm{VP}-\mathrm{HCl}$ and $\mathrm{P} 4 \mathrm{VP}-\mathrm{H}_{3} \mathrm{PO}_{4}$ were found to be reactive for primary (entries 8-9) and secondary alcohols (entries 3,6 ) as well as polyhydroxy compounds with (entries 16) longer reaction time. There were no reactions observed with phenol (entry 13). All three catalysts were reactivated after washing with dichloromethane and drying at $70{ }^{\circ} \mathrm{C}$ for 2 hours. They retained their catalytic activities up to four times in case of acetylation of cholesterol.

\section{Conclusion}

In conclusion we have demonstrated the advantages of using poly(4-vinylpyridine) supported Brønsted acids are (i) simple method of preparation, (ii) good stability, (iii) easy recovery and recycling without loss of catalytic activity (iv) safe handling, (v) good reactivity (vi) possibility of wide applications as solid acid catalyst in other organic reactions.

Acknowledgments. The authors are thankful to CSIR, New
Delhi, India for granting a Research Project No. 32-209/2006 (SR) to RB.

\section{References}

1. (a) Delmas, M.; Gaset, A. Synthesis 1980, 11, 871 (b) Aramendia, M. A.; Borau, V.; Jimenez, C.; Marinas, J. M.; Romero, F. J.; Urbano, F. J. Catal. Lett. 2001, 73, 203.

2. Benaglia, M.; Puglisi, A.; Cozzi, F. Chem. Rev. 2003, 103, 3401.

3. Barth , M.; Rademann, J. J. Comb. Chem. 2004, 6, 340.

4. Rao, V.; Ashokan, P. V.; Shridhar, M. H. Materials Science and Engineering: A 2000, 276, 266.

5. (a) Lee, B. S.; Mahajan, S.; Janda, K. D. Tetrahedron 2005, 61, 3081. (b) Friderich, H. B.; Singh, N. Cat. Lett. 2006, 110, 61. (c) Malynych, S.; Luzinov, I.; Chumanov, G. J. Phys. Chem. B 2002, 106, 1280.

6. (a) Sethna, S.; Phadke, R. Org. React. 1953, 7, 1. (b) Fehnel, E. A. J. Org. Chem. 1966, 31, 2899. (c) Biginelli, P. Gazz. Chim. Ital. 1893, 23, 360 .

7. Li, T-S.; Li, A-X. J. Chem. Soc. Perkin Trans. I 1998,1913 and references cited there in

8. Borah, K. J.; Phukan, M.; Borah, R. Synth. Commun. 2008, 38, 3082.

9. Yadav, M. K.; Kothari, A. V.; Naik, D. G.; Gupta, V. K. Green Chem. Lett \& Rev. 2009, 2, 181. 